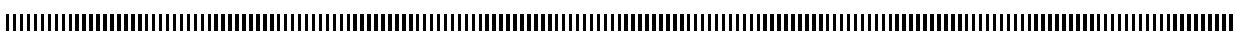

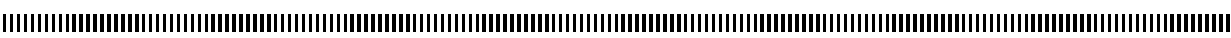

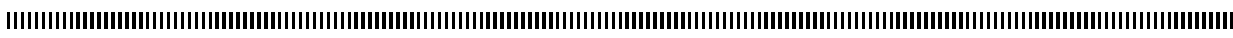

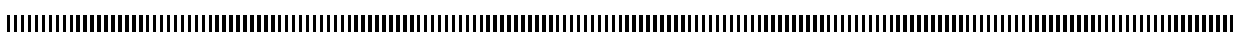

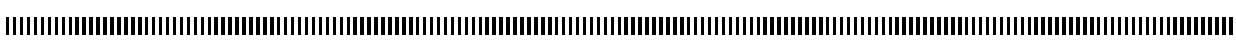
| | |

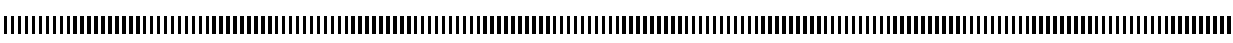
|

\title{
Nonlinear Control for Isolated DC MicroGrids
}

\author{
Filipe Perez, Gilney Damm, Françoise Lamnabhi-Lagarrigue, Paulo Ri- \\ beiro
}

L2S Laboratory / IBISC Laboratory / Institute of Electrical Systems and Energy

Université Paris-Saclay / Federal University of Itajubá

Gif-sur-Yvette / Itajubá

France / Brazil

filipe.perez@12s.centralesupelec.fr

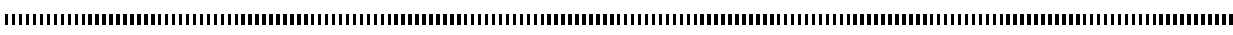

RÉSUMÉ. L'accès à l'électricité dans les communautés isolées est capital pour améliorer la vie dans ces sociétés. L'utilisation de sources d'énergie renouvelables couplés à des systèmes de stockage d'énergie est la clé pour l'approvisionnement en énergie propre dans les zones non-connectés au sans réseau principal. Le fonctionnement autonome des énergies renouvelables représente un défi pour leur exploitation et la fiabilité, par conséquent, le concept de MicroGrid DC est considéré comme une solution puissante permettant l'intégration de ces énergies renouvelables et un fonctionnement fiable du systéme de maniére simple. Cet article propose une stratégie de commande distribuée non linéaire pour un MicroGrid isolé composé d'énergies renouvelables et de différents systèmes de stockage pour alimenter la charge. Les résultats de simulation montrent le comportement du MicroGrid proposé ainsi qu' une comparaison avec le contrôle linéaire classique, de manière à mettre en évidence les performances de la commande.

ABSTRACT. The access to electricity for isolated communities is capital for improving life in those societies. The use of renewable energy sources (renewables) and energy storage systems is the key for clean energy supply in remote areas without the main grid. The standalone operation of renewables represents a challenge for operation and reliability, therefore the DC MicroGrid concept is seen as a powerful solution allowing renewables integration and reliable operation of the system in a simple way. This paper proposes a distributed nonlinear control strategy for an isolated MicroGrid composed of renewables and different time-scale storage systems to supply a DC load. The simulations results show the behavior of the proposed MicroGrid and a comparison with classical linear control is done to highlight the control performance.

MOTS-CLÉS : MicroGrids, systèmes d'alimentation isolés, contrôle non linéaire, intégration de source renouvelable, stabilité de réseau, SmartGrids

KEYWORDS : MicroGrids, isolated power systems, nonlinear control, renewable source integration, grid stability, SmartGrids

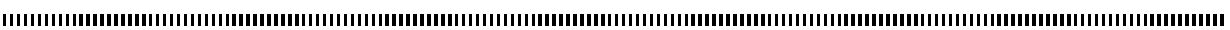




\section{Introduction}

A significant number of people in remote communities live without access to electricity. This villages may never have grid connection because of economic reasons and remoteness. On the other hand, many of isolated communities, such as African, Brazilian or island communities, have great potential for solar radiation and wind allowing the use of renewable energy sources (renewables). Electricity supply can be seen as a contribution to social inclusion and improvement of quality of life where electricity is mainly used for household purposes such as lighting, heating and others to meet local energy demand $[1,2]$. In addition, the transition for an electricity based energy usage avoids consuming local carbon based resources like coal or wood. This helps improving the conservation of ecosystems and mitigation of $\mathrm{CO}_{2}$ emissions.

The standalone grid application requires a proper operation to provide reliable, continuous, sustainable and good-quality electricity to consumers, which brings several technical challenges for renewables application. The intermittent and non-dispatchable characteristic of renewables cause great impacts related to instability and fluctuations in their energy generation. In this context, the use of energy storage systems coupled with renewables operating to properly supply a local load has brought the MicroGrid concept, which is a powerful solution to accomplish the targets of standalone grid operation, improving reliability, resilience and availability of the whole system $[3,4,5]$.

More specifically, direct current (DC) MicroGrids can be considered an advance for system efficiency and simplicity, as most storage systems, renewables and loads are inherently DC. The DC power converters have simpler topologies as well as control design for a DC MicroGrid becomes simpler and less expensive, since there is no reactive power or frequency and inertia as in alternating current (AC) [6, 7].

Reliability and stability is essential for the operation of an isolated MicroGrid. Therefore, a rigorous study of stability of the whole system is crucial. In this sense, the nonlinear control approach becomes more interesting, since it allows the use of a more realistic model and a control strategy that can work in a wider operation region. The linear control approach, widely diffused in literature and industry due to simplicity, often has a limited operation region and does not present a rigorous study of stability, so nonlinear control proves to be more advantageous and usually better performing $[8,9,10,11]$.

This paper proposes a distributed nonlinear control strategy to provide stability for a DC MicroGrid composed of photovoltaic panels (PV) as the main generation; two energy storage systems with different time-scales, the batteries with slower dynamics used to balance the power flow in the DC grid and the supercapacitor with faster dynamics to stabilize the voltage on the DC bus; a variable DC load that can represent lights and heating/cooking systems in the isolated area, which must be properly supply with constant voltage following the grid requirements. The contribution here is to develop simpler local controllers by applying nonlinear techniques compared with the proposed in [9], then the stability of the whole grid is assured, where a second level control is assumed to exist.

It is developed a control law for each device in the system, where the PV must work at the maximum power point, the battery realizes balance on power flow and the DC load have to be supplied with constant voltage. To stabilize the DC bus of the MicroGrid, a control law is applied to a bidirectional boost converter that connects the supercapacitor system to the DC grid. The control scheme is based on dynamical feedback linearization and consists in splitting the system in different time scale dynamics, creating consequently a simplified model to reduce size and complexity of the control system. The non-minimal 


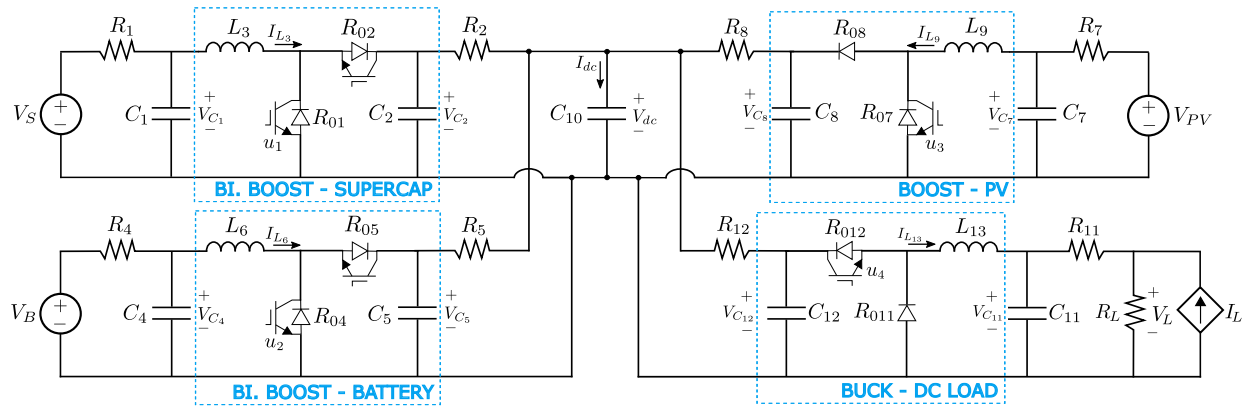

Figure 1. The considered DC MicroGrid model.

phase problem of the boost converter according to the chosen control output is solved using singular perturbation analysis based on this explicit time-scale separation.

This paper is organized as follows : Section II introduces the considered MicroGrid model, while Section III describes the nonlinear control strategy applied. Simulations results on Matlab/Simulink are outlined in Section IV and conclusions are provided in Section V.

\section{MicroGrid Model}

The considered DC MicroGrid has the target to assure voltage stability in the DC bus and properly feed power to the load while absorbing the maximum power from the PV array. Another important target is to save battery lifetime by allocating the battery to injecting/absorbing power with piecewise constant profile. Since the PV and the DC load introduces many time varying oscillations that could reduce battery life, a supercapacitor is used to stabilize the voltage on the DC bus by inject/absorb the right amount of power to balance the power flow in the grid. Power given by the battery will then be modified according to the new level of energy in the supercapacitor, and according to a desired charge/discharge rate [12]. Two assumptions are made : a higher level controller is supposed to provide references to be accomplished by the local controllers [13]; the second one is about a proper sizing of PV array, battery and supercapacitor in order to have feasible power balance with respect to the sizing of the load. The DC MicroGrid scheme is introduced in Figure 1.

By applying Kirchoff law in the MicroGrid circuit we may obtain the state space equation of the whole grid.

$$
\begin{array}{r}
\dot{V}_{C_{1}}=\frac{1}{R_{1} C_{1}} V_{S}-\frac{1}{R_{1} C_{1}} V_{C_{1}}-\frac{1}{C_{1}} I_{L_{3}} \\
\dot{V}_{C_{2}}=\frac{1}{R_{2} C_{2}} V_{d c}-\frac{1}{R_{2} C_{2}} V_{C_{2}}+\frac{1}{C_{2}} I_{L_{3}}\left(1-u_{1}\right) \\
\dot{I}_{L_{3}}=\frac{1}{L_{3}} V_{C_{1}}-\frac{1}{L_{3}} V_{C_{2}}\left(1-u_{1}\right)-\frac{R_{01}}{L_{3}} I_{L_{3}} \\
\dot{V}_{C_{4}}=\frac{1}{R_{4} C_{4}} V_{B}-\frac{1}{R_{4} C_{4}} V_{C_{4}}-\frac{1}{C_{4}} I_{L_{6}}
\end{array}
$$




$$
\begin{aligned}
& \dot{V}_{C_{5}}=\frac{1}{R_{5} C_{5}} V_{d c}-\frac{1}{R_{5} C_{5}} V_{C_{5}}+\frac{1}{C_{5}} I_{L_{6}}\left(1-u_{2}\right) \\
& \dot{I}_{L_{6}}=\frac{1}{L_{6}} V_{C_{4}}-\frac{1}{L_{6}} V_{C_{5}}\left(1-u_{2}\right)-\frac{R_{04}}{L_{6}} I_{L_{6}} \\
& \dot{V}_{C_{7}}=\frac{1}{R_{7} C_{7}} V_{P V}-\frac{1}{R_{7} C_{7}} V_{C_{7}}-\frac{1}{C_{7}} I_{L_{9}} \\
& \dot{V}_{C_{8}}=\frac{1}{R_{8} C_{8}} V_{d c}-\frac{1}{R_{8} C_{8}} V_{C_{8}}+\frac{1}{C_{8}} I_{L_{9}}\left(1-u_{3}\right) \\
& \dot{I}_{L_{9}}=\frac{1}{L_{9}} V_{C_{7}}-\frac{1}{L_{9}} V_{C_{8}}\left(1-u_{3}\right)-\frac{R_{08}}{L_{9}} I_{L_{9}}+ \\
& +\frac{1}{L_{9}}\left(R_{08}-R_{07}\right) I_{L_{9}} u_{3} \\
& \dot{V}_{C_{11}}=\frac{1}{R_{11} C_{11}} V_{L}-\frac{1}{R_{11} C_{11}} V_{C_{11}}+\frac{1}{C_{11}} I_{L_{13}} \\
& \dot{V}_{C_{12}}=\frac{1}{R_{12} C_{12}} V_{d c}-\frac{1}{R_{12} C_{12}} V_{C_{12}}-\frac{1}{C_{12}} I_{L_{13}} u_{4} \\
& \dot{I}_{L_{13}}=-\frac{1}{L_{13}} V_{C_{11}}+V_{C_{12}} u_{4}-\frac{R_{011}}{L_{13}} I_{L_{13}}+ \\
& -\frac{1}{L_{13}}\left(R_{012}-R_{011}\right) I_{L_{13}} u_{4} \\
& \dot{V}_{d c}=\frac{1}{C_{10}}\left[\frac{1}{R_{2}}\left(V_{C_{2}}-V_{d c}\right)+\frac{1}{R_{5}}\left(V_{C_{5}}-V_{d c}\right)+\right. \\
& \left.+\frac{1}{R_{8}}\left(V_{C_{8}}-V_{d c}\right)+\frac{1}{R_{12}}\left(V_{C_{12}}-V_{d c}\right)\right]
\end{aligned}
$$

The supercapacitor is selected here to quickly respond to grids oscillations caused by load variations and PV intermittent feature. Therefore, this subsystem can stabilizes the MicroGrid's transients due its fast response characteristic. A bidirectional-boost converter connects the supercapacitor in the DC link. The average state-space model of the supercapacitor subsystem is present in (1)-(3).

$V_{S}$ is the supercapacitor's voltage, $V_{C_{1}}$ is the voltage on capacitor $C_{1}, V_{C_{2}}$ is the voltage on capacitor $C_{2}, I_{L_{3}}$ is the current on inductor $L_{3} . R_{1}$ and $R_{2}$ are the resistances representing the cable losses, $R_{01}$ and $R_{02}$ is switch losses of the semiconductors and $u_{1}$ is the duty cycle. For bidirectional converters : $R_{01}=R_{02}$.

The battery system operates for slow power variations in the grid, where the current has piecewise constant variations to save its lifetime, since a battery has limited number of cycles. The main battery target is to provide power flow regulation in long term. A 
bidirectional-boost converter connects the battery in the MicroGrid. The average statespace model of the battery subsystem is written in (4)-(6).

$V_{B}$ is the battery's voltage, $V_{C_{4}}$ is the voltage on capacitor $C_{4}, V_{C_{5}}$ is the voltage on capacitor $C_{5}, I_{L_{6}}$ is the current on inductor $L_{6} . R_{4}$ and $R_{5}$ are the resistances representing the cable losses, $R_{04}$ and $R_{05}$ are switch losses of the semiconductors, where $R_{04}=R_{05}$, and $u_{2}$ is the duty cycle of the converter.

The PV array is the main power generation in the MicroGrid sized according to the MicroGrid's load. The MPPT algorithm in the second level control make the system works at the maximum power point. A boost converter is used to connect the PV into DC bus. The state-space model of the PV subsystem is presented in equations (7)-(9).

$V_{P V}$ is the panel's voltage, $V_{C_{7}}$ is the voltage on capacitor $C_{7}, V_{C_{8}}$ is the voltage on capacitor $C_{8}, I_{L_{9}}$ is the current on inductor $L_{9} . R_{7}$ and $R_{8}$ are the resistances representing the cable losses, $R_{07}$ and $R_{08}$ are switch losses of the semiconductors and $u_{3}$ is the duty cycle of the converter.

The DC load in the MicroGrid can represent basically lights and heating/cooking for an isolated MicroGrid varying the power according to the commune need. The main load requirement is to keep its voltage around of the grid requirements (normally $\pm 5 \%$ ). A buck converter connects the load into the grid and state-space model of the load subsystem is written in equations (10)-(12).

$V_{L}$ is the load voltage, $V_{C_{11}}$ is the voltage on capacitor $C_{11}, V_{C_{12}}$ is the voltage on capacitor $C_{12}, I_{L_{13}}$ is the current on inductor $L_{13} . R_{11}$ and $R_{12}$ are the resistances representing the cable losses, $R_{011}$ and $R_{012}$ are switch losses of the semiconductors and $u_{4}$ is the duty cycle of the converter. The variations in the DC load is modelled as a current source $\left(I_{L}\right)$ and can be related with the load voltage $\left(V_{L}\right)$ as follows :

$$
V_{L}=\frac{R_{L}}{R_{11} R_{L}}\left(V_{C_{11}}-R_{11} I_{L}\right)
$$

The considered second level controller is responsible to provide the references for the MicroGrid related to the condition of the whole system. Here, the reference value $I_{L_{6}}^{*}$ is provided to the battery subsystem, taking into account balance on power flow, stateof-charge of the supercapacitor and battery and the current limitation in the battery. The reference value $I_{L_{9}}^{*}$ for the PV calculated by the MPPT algorithm, which is the incremental conductance method. The DC load is supplied at constant level of voltage $V_{C_{11}}^{*}$ according to grid standards. Therefore, the local control strategy can be developed based on this hierarchical control structure $[14,15]$.

\section{Control Strategy}

This section describes the control strategy of each device of the MicroGrid and provides a control law to assure stability of the whole grid.

A "plug and play" approach based on the "system of systems" philosophy using distributed control methodologies is developed for this MicroGrid, since this kind of approach can work better due remoteness of the system. Therefore, the supercapacitor is allocated to regulate the voltage $V_{d c}$ by controlling the output voltage $V_{C_{2}}$ on its converter, which deal with the fast oscillations and transients of the DC bus. The reference for the battery is the current $I_{L_{6}}^{*}$, which is given by the second level controller to control the slower variations on the grid. The reference of the PV is current $I_{L_{9}}^{*}$ calculated by the MPPT 
algorithm to extract the maximum power from the PV array. Lastly, the control target in the DC load is maintain voltage $V_{C_{11}}$ on its desired constant value $V_{C_{11}}^{*}$.

\subsection{Supercapacitor system}

The supercapacitor control target is to regulate the DC bus voltage $\left(V_{d c}\right)$ to assure stability of its dynamic. For this, the voltage $V_{C_{2}}$ is the control input chosen to accomplish this target. Therefore, we can control $V_{C_{2}}$ by driving $V_{C_{2}}$ to the desired voltage $V_{C_{2}}^{*}$, such that, $V_{d c}$ is stabilized.

The control structure follows the consideration : the supercapacitor system (1)-(3) is composed of two subsystems, first one is the current subsystem $\left(I_{L_{3}}\right)$ and second one is the voltages subsystem $\left(V_{C_{1}}, V_{C_{2}}\right.$ and $\left.V_{d c}\right)$. There is a control law, such that the current subsystem is allocated to have much faster dynamics than voltages subsystem. Therefore, by properly choosing the convergence speed of both subsystems we create the singular perturbation condition based on explicit time-scale separation.

Since $I_{L_{3}}$ will be controlled to have much faster dynamics than the voltages, under some mild conditions the controllers can be developed separately [16]. First, we propose the control design to steer $I_{L_{3}} \rightarrow I_{L_{3}}^{*}$, where $I_{L_{3}}^{*}$ is yet to be designed. Thus, $I_{L_{3}}^{*}$ will be deduced based on a simplified model for $V_{C_{2}}$ obtained by singular perturbation result, that can steer $V_{C_{2}}$ to $V_{C_{2}}^{*}$, where $V_{C_{2}}^{*}$ is calculated to stabilize the DC link in the MicroGrid.

\subsubsection{Current subsystem control law}

The first step is to control the current $I_{L_{3}}$ to its reference $I_{L_{3}}^{*}$. According to the control objective, let us define the output as $y_{1}=I_{L_{3}}$. It is considered that the system has one output and one input, thus a square system is shown in (15).

$$
\left\{\begin{array}{l}
\dot{I}_{L_{3}}=f_{1}\left(V_{C_{1}}, V_{C_{2}}, I_{L_{3}}\right)+g_{1} u_{1} \\
y_{1}=I_{L_{3}}
\end{array}\right.
$$

The Lie derivative of the output with respect to $g_{1}$ is :

$$
J_{1}=-\frac{V_{C_{2}}}{L_{3}}
$$

$J_{1}$ is non-singular since $V_{C_{2}}$ is always positive by technological reasons, therefore a nonlinear feedback linearizing control input can be written as :

$$
\begin{gathered}
u_{1}=J_{1}^{-1}\left[v_{3}-\frac{1}{L_{3}}\left(V_{C_{1}}-V_{C_{2}}-R_{01} I_{L_{3}}\right)\right] \\
u_{1}=1+\frac{1}{V_{C_{2}}}\left[L_{3} v_{3}-V_{C_{1}}+R_{01} I_{L_{3}}\right]
\end{gathered}
$$

where $v_{3}$ is the additional input to be designed.

Since $I_{L_{3}}^{*}$ is the desired trajectory for $I_{L_{3}}$, it is possible to design the additional input $v_{3}$ in a linear manner with respect to the output $I_{L_{3}}$ and hence a linear stable subspace is generated.

$$
\begin{aligned}
& v_{3}=\dot{I}_{L_{3}}^{*}-K_{3}\left(I_{L_{3}}-I_{L_{3}}^{*}\right)-K_{3}^{\alpha} \alpha_{3} \\
& \dot{\alpha}_{3}=I_{L_{3}}-I_{L_{3}}^{*}
\end{aligned}
$$

where $K_{3}$ and $K_{3}^{\alpha}$ are positive constants.

$v_{3}$ is the desired dynamics of $I_{L_{3}}$ and $\alpha_{3}$ an auxiliary variable to represent a integral term. This can be interpreted as a PI controller from linear control theory. 


\subsubsection{Voltages subsystem control law}

Now, the crucial step is to properly calculate the reference value $I_{L_{3}}^{*}$, such that $V_{C_{2}} \rightarrow$ $V_{C_{2}}^{*}$. Here, the task is to design a slowly varying $I_{L_{3}}^{*}$ whose derivative has minimum effect in the current control designed previously.

From singular perturbation analysis, we can consider that $I_{L_{3}}$ is already $I_{L_{3}}^{*}$ on $V_{C_{2}}$ dynamics because of time-scale separation $\left(I_{L_{3}}\right.$ is considered much faster than $\left.V_{C_{2}}\right)$. Therefore, we may replace $V_{C_{2}}$ dynamics as follows $[17,18,19,20]$.

$$
\dot{V}_{C_{2}}=\frac{1}{R_{2} C_{2}} V_{d c}-\frac{1}{R_{2} C_{2}} V_{C_{2}}+\frac{1}{C_{2}} I_{L_{3}}^{*}\left(1-u_{1}^{*}\right)
$$

where :

$$
u_{1}^{*}=1-\frac{1}{V_{C_{2}}}\left[V_{C_{1}}-R_{01} I_{L_{3}}^{*}\right]
$$

Therefore, the result is a simplified model of $V_{C_{2}}$ dynamics, which can be written as :

$$
\dot{V}_{C_{2}}=\frac{1}{R_{2} C_{2}} V_{d c}-\frac{1}{R_{2} C_{2}} V_{C_{2}}+\frac{I_{L_{3}}^{*}}{C_{2} V_{C_{2}}}\left[V_{C_{1}}-R_{01} I_{L_{3}}^{*}\right]
$$

The singular perturbation result is a simplified model of $V_{C_{2}}$, then a good choice for the new control input is take $: v_{d}=\dot{I}_{L_{3}}^{*}$, called dynamical feedback approach. With $v_{d}$ as the new control input we have full state transformation with no zero dynamics. Besides that, we can easily find the control reference $I_{L_{3}}^{*}$ by integrating the new control input $I_{L_{3}}^{*}=\int v_{d} d t$.

Applying input/output feedback linearization theory and defining $V_{C_{2}}$ as the control output, the relative degree is 2 . Consequently, we may find $v_{d}$ in the second time-derivative of the simplified $V_{C_{2}}$ dynamics. The following Lie derivatives can be deduced as :

$$
\begin{gathered}
\dot{V}_{C_{2}}=L_{f_{2}}^{1} h_{2}(x) \\
\ddot{V}_{C_{2}}=\frac{\dot{V}_{d c}}{R_{2} C_{2}}-\dot{V}_{C_{2}}\left[\frac{1}{R_{2} C_{2}}+\frac{V_{C_{1}} I_{L_{3}}^{*}-R_{01} I_{L_{3}}^{*}{ }^{2}}{C_{2} V_{C_{2}}{ }^{2}}\right]+ \\
+\frac{1}{C_{2} V_{C_{2}}} I_{L_{3}}^{*} \dot{V}_{C_{1}}+\frac{1}{C_{2} V_{C_{2}}}\left(V_{C_{1}}-2 R_{01} I_{L_{3}}^{*}\right) \dot{I}_{L_{3}}^{*}
\end{gathered}
$$

Therefore, we have found the control input $v_{d}$ as follows :

$$
\ddot{V}_{C_{2}}=L_{f_{2}}^{2} h_{2}(x)+L_{g_{2}} L_{f_{2}}^{1} h_{2}(x) v_{d}
$$

By introducing a synthetic input $\theta_{d}$, the input $v_{d}$ can be designed as :

$$
v_{d}=\frac{1}{L_{g_{2}} L_{f_{2}}^{1} h_{2}(x)}\left[\theta_{d}-L_{f_{2}}^{2} h_{2}(x)\right]
$$

The synthetic input is chosen using linear techniques to give the desired dynamics for $V_{C_{2}}$.

$$
\theta_{d}=-K_{2}\left(\dot{V}_{C_{2}}-\dot{V}_{C_{2}}^{*}\right)+K_{2}^{\alpha}\left(V_{C_{2}}-V_{C_{2}}^{*}\right)
$$


By substituting (25) and (26) in (21) and (24), and considering $\dot{V}_{C_{2}}^{*}=0$ since reference $V_{C_{2}}^{*}$ will be given by a constant reference value, the following closed-loop is obtained :

$$
\left[\begin{array}{c}
\dot{V}_{C_{2}} \\
\ddot{V}_{C_{2}}
\end{array}\right]=\left[\begin{array}{cc}
0 & 1 \\
-K_{2}^{\alpha} & -K_{2}
\end{array}\right]\left[\begin{array}{c}
V_{C_{2}}-V_{C_{2}}^{*} \\
\dot{V}_{C_{2}}
\end{array}\right]
$$

where we can define the speed of convergence for $V_{C_{2}}$, using $K_{2}$ and $K_{2}^{\alpha}$ by pole placement to get slower voltage dynamics for the simplified model.

Remark 1 : One of the tasks is to design a slowly varying $I_{L_{3}}^{*}$ whose derivative has negligible effects on the current subsystem.

Remark 2 : The use of singular perturbation to obtain the simplified model is valid inside an operating region that is given by the time scale ratio from the two subsystems. As a consequence, the desired imposed dynamics should be chosen such as to obtain a suitable operation region for the DC MicroGrid.

Remark 3 : In the proposed control law, the dynamical feedback linearization approach is chosen since we get full state transformation achieving a linear second-order system.

\subsection{Battery system}

The reference value $I_{L_{6}}^{*}$ for the battery system is given by second level controller, which is related to the power flow regulation and state of charge of the supercapacitor. According to the control objective, let us define the control output as $y_{2}=I_{L_{6}}$. It is considered that the system has one output and one input, thus a square system is shown in (27).

$$
\left\{\begin{array}{l}
\dot{I}_{L_{6}}=f_{6}\left(V_{C_{4}}, V_{C_{5}}, I_{L_{6}}\right)+g_{6} u_{2} \\
y_{2}=I_{L_{6}}
\end{array}\right.
$$

The Lie derivative of the output with respect to $g_{6}$ is :

$$
J_{2}=-\frac{V_{C_{5}}}{L_{6}}
$$

$J_{2}$ is non-singular since the voltage $V_{C_{5}}$ is always positive, therefore a nonlinear feedback control input can be written as :

$$
\begin{gathered}
u_{2}=J_{2}^{-1}\left[v_{6}-\frac{1}{L_{6}}\left(V_{C_{4}}-V_{C_{5}}-R_{04} I_{L_{6}}\right)\right] \\
u_{2}=1+\frac{1}{V_{C_{5}}}\left[L_{6} v_{6}-V_{C_{4}}+R_{04} I_{L_{6}}\right]
\end{gathered}
$$

where $u_{2}$ is the control input in (27) and $v_{6}$ is the additional input.

Since $I_{L_{6}}^{*}$ is the desired trajectory for $I_{L_{6}}$, it is possible to design the additional input $v_{6}$ in a linear manner with respect to the output $I_{L_{6}}$ and hence a linear stable subspace is generated.

$$
\begin{aligned}
& v_{6}=\dot{I}_{L_{6}}^{*}-K_{6}\left(I_{L_{6}}-I_{L_{6}}^{*}\right)-K_{6}^{\alpha} \alpha_{6} \\
& \dot{\alpha}_{6}=I_{L_{6}}-I_{L_{6}}^{*}
\end{aligned}
$$

where $K_{6}$ and $K_{6}^{\alpha}$ are positive constants and $\alpha_{6}$ is an integral term. 


\section{Zero dynamics}

The states $V_{C_{4}}$ and $V_{C_{5}}$ are the zero dynamics for the battery subsystem. The calculated equilibrium points are given by :

$$
\begin{gathered}
V_{C_{4}}^{e}=V_{B}-R_{4} I_{L_{6}}^{*} \\
V_{C_{5}}^{e}=\frac{V_{d c}}{2} \pm \frac{1}{2} \sqrt{V_{d c}^{2}+4 R_{5} I_{L_{6}}^{*}\left(V_{C_{4}}-R_{04} I_{L_{6}}^{*}\right)}
\end{gathered}
$$

To analyze the zero dynamics stability, the Jacobian linearization matrix $B$ is presented bellow :

$$
B=\left[\begin{array}{cc}
-\frac{1}{R_{4} C_{4}} & 0 \\
\frac{1}{C_{5}} \frac{I_{L_{6}}^{*}}{V_{C_{5}}} & -\frac{1}{R_{5} C_{5}}-\frac{I_{L_{6}}^{*}\left(V_{C_{4}}-R_{04} I_{L_{6}}^{*}\right)}{C_{5} V_{C_{5}}^{e}{ }^{2}}
\end{array}\right]
$$

The eigenvalues $\lambda$ can be written as :

$$
\lambda_{1,2}=-\frac{a}{2} \pm \frac{1}{2} \sqrt{a^{2}-4 b}
$$

where :

$$
\begin{aligned}
& a=\frac{1}{R_{4} C_{4}}+\frac{1}{R_{5} C_{5}}+\frac{I_{L_{6}}^{*}}{C_{5} V_{C_{5}}^{e}{ }^{2}}\left(V_{C_{4}}-R_{04} I_{L_{6}}^{*}\right) \\
& b=\frac{1}{R_{4} C_{4}}\left[\frac{1}{R_{5} C_{5}}+\frac{I_{L_{6}}^{*}}{C_{5} V_{C_{5}}^{e}}\left(V_{C_{4}}-R_{04} I_{L_{6}}^{*}\right)\right]
\end{aligned}
$$

Stability of the equilibrium points will depends on the sign of $a$ and $b$, then the following stability analysis is made.

1) For $a>0$ and $b>0$ : the eigenvalues is $\operatorname{Re}\left[\lambda_{1,2}<0\right]$, consequently the equilibrium points of the zero dynamics are stable. Working on the above inequalities, we find out a region related to $I_{L_{6}}^{*}$. The first region is given by $a>0$ :

$$
\frac{V_{C_{4}}-\sqrt{\Delta_{3}}}{2 R_{04}}<I_{L_{6}}^{*}<\frac{V_{C_{4}}+\sqrt{\Delta_{3}}}{2 R_{04}}
$$

where :

$$
\Delta_{3}={V_{C_{4}}}^{2}+4 R_{04} C_{5} V_{C_{5}}^{e}\left[\frac{1}{R_{4} C_{4}}+\frac{1}{R_{5} C_{5}}\right]
$$

The second region given by $b>0$ can be expressed as :

$$
\frac{V_{C_{4}}-\sqrt{\Delta_{4}}}{2 R_{04}}<I_{L_{6}}^{*}<\frac{V_{C_{4}}+\sqrt{\Delta_{4}}}{2 R_{04}}
$$

with :

$$
\Delta_{4}=V_{C_{4}}^{2}+\frac{4 R_{04}}{R_{5}} V_{C_{5}}^{e}
$$

The intersection of (37) and (38) is actually given by (38), which is the region of stability for $I_{L_{6}}^{*}$, where zero dynamics are locally stable. 
2) For $a \geq 0$ and $b \leq 0$ : at least one eigenvalue will have a positive real part $\operatorname{Re}\left[\lambda_{1,2} \geq 0\right]$, then the system is not stable.

3) For $a \leq 0$ : we also have at least one eigenvalue with positive real part $\operatorname{Re}\left[\lambda_{1,2} \geq 0\right]$ where the zero dynamics are not stable.

The control of $I_{L_{6}} \rightarrow I_{L_{6}}^{*}$ is stable to charge and discharge battery according to the regions described by the introduced analysis.

\subsection{PV array}

The incremental conductance MPPT algorithm is based on the calculation of the power derivative in the characteristic curve of the panel to find the maximum of this function. Then, this value is given by a reference current $I_{L_{9}}^{*}$ for the PV array to work at the maximum power point. According to the control objective, let us define the control output as $y_{3}=I_{L_{9}}$. It is considered that the system has one output and one input, thus a square system is shown in (39).

$$
\left\{\begin{array}{l}
\dot{I}_{L_{9}}=f_{9}\left(V_{C_{7}}, V_{C_{8}}, I_{L_{9}}\right)+g_{9} u_{3} \\
y_{3}=I_{L_{9}}
\end{array}\right.
$$

The Lie derivative of the output with respect to $g_{9}$ is :

$$
J_{3}=-\frac{V_{C_{8}}-\left(R_{08}-R_{07}\right) I_{L_{9}}}{L_{9}}
$$

$J_{3}$ is non-singular since $V_{C_{8}} \neq\left(R_{08}-R_{07}\right) I_{L_{9}}$, therefore a nonlinear feedback control input can be written as :

$$
\begin{gathered}
u_{3}=J_{3}^{-1}\left[v_{9}-\frac{1}{L_{9}}\left(V_{C_{7}}-V_{C_{8}}-R_{08} I_{L_{9}}\right)\right] \\
u_{3}=\frac{L_{9} v_{9}-V_{C_{7}}+V_{C_{8}}+R_{08} I_{L_{9}}}{V_{C_{8}}-\left(R_{08}-R_{07}\right) I_{L_{9}}}
\end{gathered}
$$

where $u_{3}$ is the control input in (39) and $v_{9}$ is the additional input.

Since $I_{L_{9}}^{*}$ is the desired trajectory for $I_{L_{3}}$, it is possible to design the additional input $v_{9}$ in a linear manner with respect to the output $I_{L_{9}}$ and hence a linear stable subspace is generated.

$$
\begin{aligned}
& v_{9}=\dot{I}_{L_{9}}^{*}-K_{9}\left(I_{L_{9}}-I_{L_{9}}^{*}\right)-K_{9}^{\alpha} \alpha_{9} \\
& \dot{\alpha_{9}}=I_{L_{9}}-I_{L_{9}}^{*}
\end{aligned}
$$

where $K_{9}$ and $K_{9}^{\alpha}$ are positive constants.

\section{Zero dynamics}

The states $V_{C_{7}}$ and $V_{C_{8}}$ are the zero dynamics in the PV system. The calculated equilibrium points are given by :

$$
\begin{gathered}
V_{C_{7}}^{e}=V_{P V}-R_{7} I_{L_{9}}^{*} \\
V_{C_{8}}^{e}=\frac{V_{d c}}{2} \pm \frac{1}{2} \sqrt{V_{d c}^{2}+4 R_{7} I_{L_{9}}^{*}\left(V_{C_{7}}-R_{07} I_{L_{9}}^{*}\right)}
\end{gathered}
$$


To analyze the zero dynamics stability, the Jacobian linearization matrix $C$ is presented bellow :

$$
C=\left[\begin{array}{cc}
-\frac{1}{R_{7} C_{7}} & 0 \\
\frac{-I_{L_{9}}^{*}}{V_{C_{8}}^{e}-\left(R_{08}-R_{07}\right) I_{L_{9}}^{*}} & -\frac{1}{R_{8} C_{8}}-\frac{I_{L_{9}}^{*}\left[V_{C_{7}}-\left(2 R_{08}-R_{07}\right) I_{L_{9}}^{*}\right]}{C_{8}\left[V_{C_{8}}^{e}-\left(R_{08}-R_{07}\right) I_{L_{9}}^{*}\right]^{2}}
\end{array}\right]
$$

In this case, we have that $I_{L_{9}} \geq 0$ is always positive, as a consequence $V_{P V} \geq V_{C_{7}}$. Therefore, we have only two possible cases in the matrix $C$ parameters :

1) For $V_{C_{7}}-\left(2 R_{08}-R_{07}\right) I_{L_{9}}^{*}<0$, the following inequality is required to obtain local stability :

$$
-\frac{1}{R_{8} C_{8}}-\frac{I_{L_{9}}^{*}\left[V_{C_{7}}-\left(2 R_{08}-R_{07}\right) I_{L_{9}}^{*}\right]}{C_{8}\left[V_{C_{8}}^{e}-\left(R_{08}-R_{07}\right) I_{L_{9}}^{*}\right]^{2}}<0
$$

with the above inequality it is possible to find the stability region for $I_{L_{9}}^{*}$, where the eigenvalues of matrix $C$ are negative and then local stability is assured.

2) For $V_{C_{7}}-\left(2 R_{08}-R_{07}\right) I_{L_{9}}^{*}>0$, the eigenvalues of matrix $C$ is always negative, then the zero dynamics is locally stable.

\subsection{DC load system}

The DC load must have a constant voltage supply, then the reference value $V_{C_{11}}^{*}$ is provided according to the grid standards. Here, we have the output control given by $y_{4}=$ $V_{C_{11}}$, then the relative degree is 2 . The DC load model can be represented as follows :

$$
\left\{\begin{array}{l}
\dot{V}_{C_{11}}=f_{11}\left(V_{L}, V_{C_{11}}\right)+g_{11} I_{L_{13}} \\
\dot{I}_{I_{13}}=f_{13}\left(V_{C_{11}}, V_{C_{12}}, V_{d c}\right)+g_{13}\left(V_{C_{12}}, I_{L_{13}}\right) u_{4} \\
y_{4}=V_{C_{11}}
\end{array}\right.
$$

Using backstteping techniques, we derivate the control output $V_{C_{11}}$ until find the control input $u_{4}$. The following Lie derivatives can be deduced as :

$$
\begin{gathered}
\dot{V}_{C_{11}}=L_{f_{11}}^{1} h_{11}(x) \\
\ddot{V}_{C_{11}}=-\frac{1}{R_{11} C_{11}}\left[\frac{1}{R_{11} C_{11}}\left(V_{L}-V_{C_{11}}\right)+\frac{1}{C_{11}} I_{L_{13}}\right]+ \\
+\frac{1}{L_{13} C_{11}}\left[-V_{C_{11}}-R_{011} I_{L_{13}}+\left[V_{C_{12}}-\left(R_{012}-R_{011}\right) I_{L_{13}}\right] u_{4}\right] \\
\ddot{V}_{C_{11}}=L_{f_{11}}^{2} h_{11}(x)+L_{g_{11}} L_{f_{11}}^{1} h_{11}(x) u_{4}
\end{gathered}
$$

By introducing a synthetic input $v_{11}$, the input $u_{4}$ can be designed as :

$$
u_{4}=\frac{1}{L_{g_{11}} L_{f_{11}}^{1} h_{11}(x)}\left[v_{11}-L_{f_{11}}^{2} h_{11}(x)\right]
$$

where the synthetic input is chosen using linear techniques to give the desired dynamics for $V_{C_{11}}$.

$$
v_{11}=-K_{11}\left(\dot{V}_{C_{11}}-\dot{V}_{C_{11}}^{*}\right)-K_{11}^{\alpha}\left(V_{C_{11}}-V_{C_{11}}^{*}\right)
$$

where $K_{11}$ and $K_{11}^{\alpha}$ are positive constants. 


\section{Zero dynamics}

The state $V_{C_{12}}$ is the zero dynamics here, where the local stability analysis is given.

$$
\dot{V}_{C_{12}}=\frac{1}{R_{12} C_{12}} V_{d c}-\frac{1}{R_{12} C_{12}} V_{C_{12}}-\frac{1}{C_{12}} I_{L_{13}}^{*} u_{4}^{*}
$$

where $I_{L_{13}}^{*}=\frac{1}{R_{11}}\left(V_{C_{11}}^{*}-V_{L}\right)$ and :

$$
u_{4}^{*}=\frac{V_{C_{11}}^{*}+R_{011} I_{L_{13}}^{*}}{V_{C_{12}}-\left(R_{012}-R_{011}\right) I_{L_{13}}^{*}}
$$

To analyze the dynamics above, let us consider its linearization. The needed equilibrium points can be determined as follows.

$$
V_{C_{12}}^{e}=\frac{-a_{12}}{2} \pm \frac{1}{2} \sqrt{a_{12}^{2}+4 \Delta_{12}}
$$

where :

$$
\begin{gathered}
a_{12}=V_{d c}-\left(R_{012}-R_{011}\right) I_{L_{13}}^{*} \\
\Delta_{12}=I_{L_{13}}^{*}\left[\left(R_{012}-R_{011}\right) V_{d c}+R_{12}\left(V_{C_{11}}+R_{011} I_{L_{13}}^{*}\right)\right]
\end{gathered}
$$

The linearization of the $V_{C_{12}}$ dynamical equation is obtained with the Jacobian equation in (55).

$$
J_{12}=-\frac{1}{R_{12} C_{12}}+\frac{I_{L_{13}}^{*}}{C_{12}}\left[\frac{V_{C_{11}}^{*}+R_{011} I_{L_{13}}^{*}}{\left[V_{C_{12}}-\left(R_{012}-R_{011}\right) I_{L_{13}}^{*}\right]^{2}}\right]
$$

In the DC load case, we have that $I_{L_{13}}^{*} \geq 0$ (sense adopted in modelling from Figure 1 ), as a consequence we can also state that $V_{C_{11}} \geq V_{L}$ always. Let us now take into account all possible cases :

1) For $V_{C_{11}}^{*}+R_{011} I_{L_{13}}^{*}<0$, we may result in :

$$
V_{C_{11}}^{*}<\frac{R_{011}}{R_{11}+R_{011}} V_{L}
$$

which is not physically possible because $V_{C_{11}} \geq V_{L}$, then this case is not considered. stability :

2) For $V_{C_{11}}^{*}+R_{011} I_{L_{13}}^{*}>0$, the following inequality is required to obtain local

$$
-\frac{1}{R_{12} C_{12}}+\frac{I_{L_{13}}^{*}}{C_{12}}\left[\frac{V_{C_{11}}^{*}+R_{011} I_{L_{13}}^{*}}{\left[V_{C_{12}}-\left(R_{012}-R_{011}\right) I_{L_{13}}^{*}\right]^{2}}\right]<0
$$

Considering inequality (57) it is possible to find the region of stability for $I_{L_{13}}^{*}$, where Jacobionan $J_{12}$ is negative assuring local stability for zero dynamics. 


\subsection{DC bus stability analysis}

Different from previous results (see [9]), here the fixed reference value $V_{d c}^{*}$ is used as the desired reference for $V_{C_{2}}$, which means steer $V_{C_{2}} \rightarrow V_{d c}^{*}$. Then, instead of a timevarying $V_{C_{2}}^{*}$, the voltage $V_{C_{2}}$ is tracked in a fixed reference value : $V_{C_{2}}^{*}=V_{d c}^{*}$. This will induce a small steady state error in $V_{d c}$, which is the voltage drop in $R_{2}$ (cable loss). Then, the resulting value of the DC bus voltage can be written as :

$$
V_{d c}=V_{d c}^{*}-R_{2} I_{L_{3}}^{*}
$$

The result is a very convenient simplification on the control design where the operation of the MicroGrid is not affected, but limited in the operation bounds of the grid requirements, in this case $\pm 5 \%$.

The behavior of $V_{d c}$ dynamics must be analyzed to verify its convergence. Then, $V_{d c}$ can be seen as zero dynamics calculated as follows :

$$
\begin{aligned}
\dot{V}_{d c}=\frac{1}{C_{10}} & {\left[\frac{1}{R_{2}}\left(V_{d c}^{*}-V_{d c}\right)+\frac{1}{R_{5}}\left(V_{C_{5}}^{e}-V_{d c}\right)+\right.} \\
+ & \left.\frac{1}{R_{8}}\left(V_{C_{8}}^{e}-V_{d c}\right)+\frac{1}{R_{12}}\left(V_{C_{12}}^{e}-V_{d c}\right)\right]
\end{aligned}
$$

where $V_{C_{5}}^{e}, V_{C_{8}}^{e}$ and $V_{C_{12}}^{e}$ are the equilibrium points of $V_{C_{5}}, V_{C_{8}}$ and $V_{C_{12}}$ respectively.

The equilibrium point of $V_{d c}$ is calculated to show its steady-state value, which varies according to the other devices of the MicroGrid (PV, battery and DC load).

$$
V_{d c}^{e}=\frac{1}{\frac{1}{R_{2}}+\frac{1}{R_{5}}+\frac{1}{R_{8}}+\frac{1}{R_{12}}}\left[\frac{V_{d c}^{*}}{R_{2}}+\frac{V_{C_{5}}^{e}}{R_{5}}+\frac{V_{C_{8}}^{e}}{R_{8}}+\frac{V_{C_{12}}^{e}}{R_{12}}\right]
$$

When the power flow is balanced, which means $I_{L_{3}}^{*}=0$, the equilibrium value of the DC bus voltage is $V_{d c}^{e}=V_{d c}^{*}$. To analyze the local convergence of $V_{d c}$ dynamics, let us consider its Jacobian linearization $J_{10}$.

$$
J_{10}=-\left[\frac{1}{R_{2}}+\frac{1}{R_{5}}+\frac{1}{R_{8}}+\frac{1}{R_{12}}\right]
$$

Indeed, the Jacobian $J_{10}$ is always negative, independent of the operation state of the system or another system' variables. Then, we can conclude that the equilibrium point of $V_{d c}$ is locally stable. The proposed control law is proved to assure stability of the whole grid considering the stability analysis of each dynamics of the system.

\section{Simulation Results}

The DC MicroGrid was built using SimPowerSystem toolbox from Matlab/Simulink, where a realistic model can be developed including the control law of the system. The simulations are focused to show the transient behavior of the dynamics of the system and the proposed nonlinear control performance. The time range for simulations is 10 seconds where it can be seen the supercapacitor working to keep the stability of the DC bus. Table 1 presents the element parameters of the MicroGrid.

The nominal voltage of DC bus in the MicroGrid is $630 \mathrm{~V}$, with $\pm 5 \%$ of operation bounds limitation to attend the grid requirements. Then, upper bound is $661.5 \mathrm{~V}$ and 
Table 1. MicroGrid parameters

\begin{tabular}{c|c|c|c|c} 
Supercap & Battery & PV & Load & Value \\
\hline$R_{1}$ & $R_{4}$ & $R_{7}$ & $R_{11}$ & $0.1 \Omega$ \\
\hline$C_{1}$ & $C_{4}$ & $C_{7}$ & $C_{11}$ & $10 \mathrm{mF}$ \\
\hline$R_{2}$ & $R_{5}$ & $R_{8}$ & $R_{12}$ & $0.1 \Omega$ \\
\hline$C_{2}$ & $C_{5}$ & $C_{8}$ & $C_{12}$ & $10 \mathrm{mF}$ \\
\hline$L_{3}$ & $L_{6}$ & $L_{9}$ & $L_{13}$ & $3.3 \mathrm{mH}$ \\
\hline$R_{01}$ & $R_{04}$ & $R_{07}$ & $R_{011}$ & $10 \mathrm{~m} \Omega$ \\
\hline$R_{02}$ & $R_{05}$ & $R_{08}$ & $R_{012}$ & $10 \mathrm{~m} \Omega$
\end{tabular}

the lower bound is $598.5 \mathrm{~V}$. The supercapacitor has $100 \mathrm{~F}$ of capacitance, 2, $450 \mathrm{Wh}$ of energy capacity and $420 \mathrm{~V}$ of nominal voltage. The battery is a lithium-ion one with nominal voltage of $380 \mathrm{~V}$ and $190 \mathrm{~kW} / 380 \mathrm{kWh}$ of storage capacity. The PV array is composed 1, 500 panels of $60 \mathrm{~W}$, which results in $90 \mathrm{kWp}$ of nominal power and $331.5 \mathrm{~V}$ of open-circuit voltage. The DC load is time-varying and can represent the aggregation of all load in the system, with maximum power demand of $40 \mathrm{~kW}$.

It is important to design the MicroGrid in order to always supply the load requested power. Here, the PV array is sized according to the energy consumption of the load during the full day. To make the MicroGrid independent of weather conditions, the battery should be able of supply power to the load (in a worst case scenario) during an arbitrary period of time, given according to the desired reliability of the grid. Finally, the supercapacitor must provide power to the MicroGrid during a specific time interval, dealing with the strongest power peak variation in the grid $[9,21]$. The sizing equations for this DC MicroGrid are presented as follows :

$$
\begin{gathered}
\int_{0}^{d a y} P_{P V} d t \geq \int_{0}^{d a y} P_{L} d t \\
\int_{0}^{T_{1}} P_{B} d t \geq \int_{0}^{T_{1}} P_{L} d t \\
\int_{0}^{T_{2}} P_{S} d t \geq \int_{0}^{T_{2}}\left(\Delta P_{P V}+\Delta P_{L}-\Delta P_{B}\right) d t
\end{gathered}
$$

where $T_{1}$ is the considered time interval for battery reliability and $T_{2}$ is the time step duration for the supercapacitor considering the transient variations in the grid. $\Delta P_{P V}$ and $\Delta P_{L}$ are the worst case power variations the $\mathrm{PV}$ and the load can produce, and $\Delta P_{B}$ the allowed battery's power variation.

Figure 2 presents the incident irradiation in the PV and the demanded current in the DC load respectively. The irradiation profile will induce variations in the PV power generation, as the power demand variations cause disturbances on the DC load current profile, therefore both parameters bring many time-varying disturbance to the MicroGrid. The current in the DC load is negative to represent the power absorption from the DC bus of the grid.

Figure 3 presents the voltage on supercapacitor, battery and PV array respectively. The supercapacitor voltage varies according to the absorption/injection of power into the grid. The same behavior in the battery voltage can be observed, proving the power flow balance in the MicroGrid. The PV voltage varies according to the irradiation profile.

Figure 4 presents voltages $V_{C_{1}}, V_{C_{4}}$ and $V_{C_{7}}$ respectively, which are the input voltage of their converters. Voltage $V_{C_{1}}$ is related to the power exchange between the supercapacitor and the DC bus. Voltage $V_{C_{4}}$ has the same relation with the DC bus, but is related to the battery, which realizes the power flow regulation complementing the supercapacitor's duty. The errors from the references given by the second level controller are dealt by the 


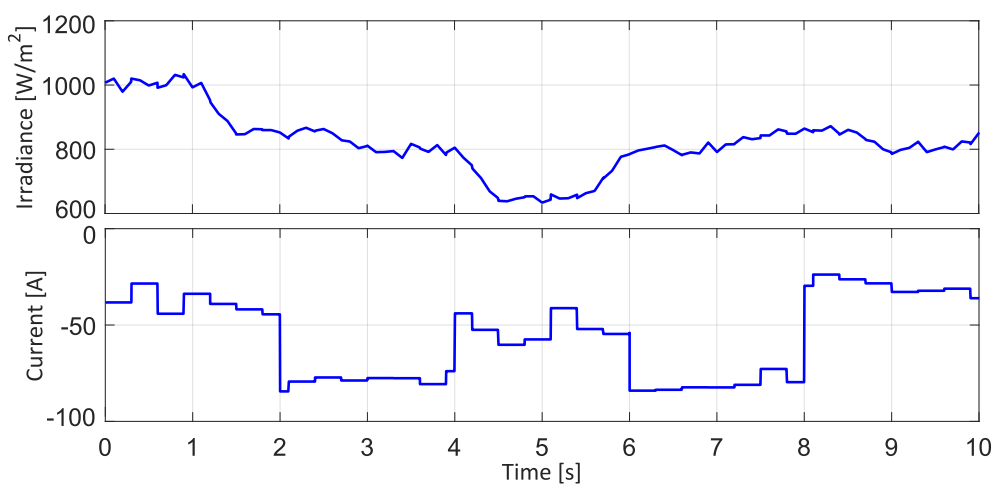

Figure 2. The incident irradiation on $P V$ and the demanded current on $D C$ load.
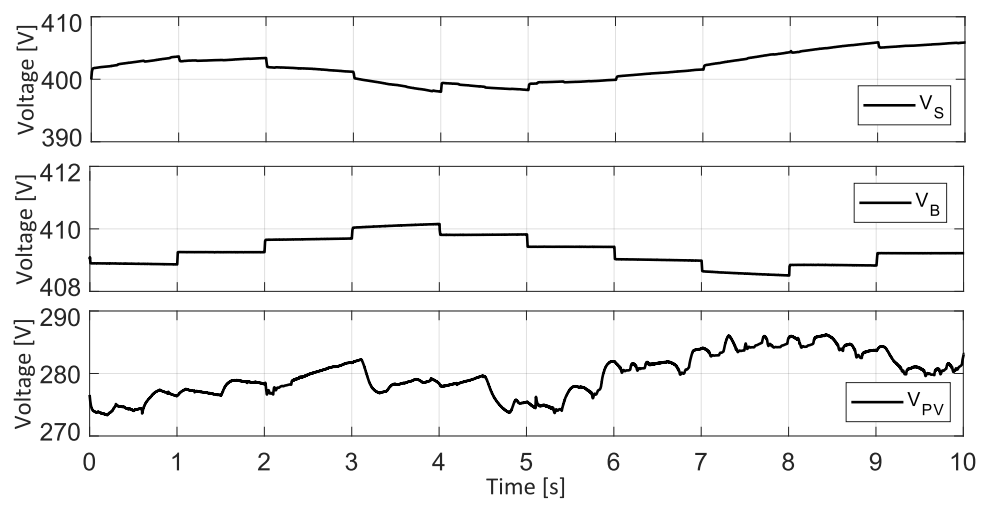

Figure 3. Voltage on supercapacitor $V_{S}$, battery $V_{B}$ and $P V$ array $V_{P V}$ respectively. supercapacitor. Voltage $V_{C_{7}}$ is related to the power generated in the PV, which highlights the interment feature of this renewable energy source.

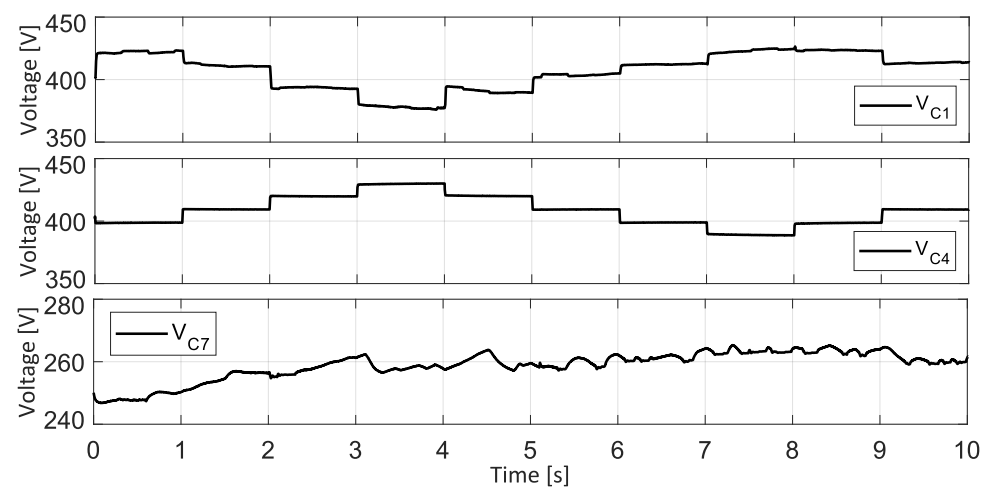

Figure 4. The voltage dynamics on $V_{C_{1}}, V_{C_{4}}$ and $V_{C_{7}}$ respectively.

Figure 5 presents the controlled voltage on DC load $\left(V_{C_{11}}\right)$ and its reference. The voltage is controlled with fast control response and the highest peaks represents variations about $0.6 \%$, which is inside the grid requirements $( \pm 5 \%)$. In conclusion, the MicroGrid 
control accomplish the target to correctly feed the DC load with good control performance.

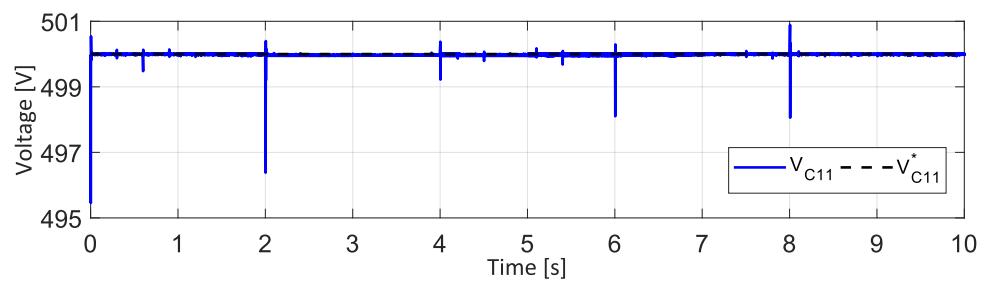

Figure 5. The controlled voltage on the $D C$ load and its reference.

Figure 6 presents the controlled currents on supercapacitor $I_{L_{3}}$ and battery $I_{L_{6}}$. Current $I_{L_{3}}$ is given by the demanded current in the DC minus the current flowing in the battery. Here, it is possible to highlight the different duty between supercapacitor and battery, since the profile of the supercapacitor current $I_{L_{3}}$ present more variations compared with battery current $I_{L_{6}}$. This because the supercapcitor's duty is to regulate the voltage on DC bus compensating the mismatch of power on $V_{d c}$, and the battery current is based on long term power flow stabilization from higher level controller. The currents track their reference with good performance.

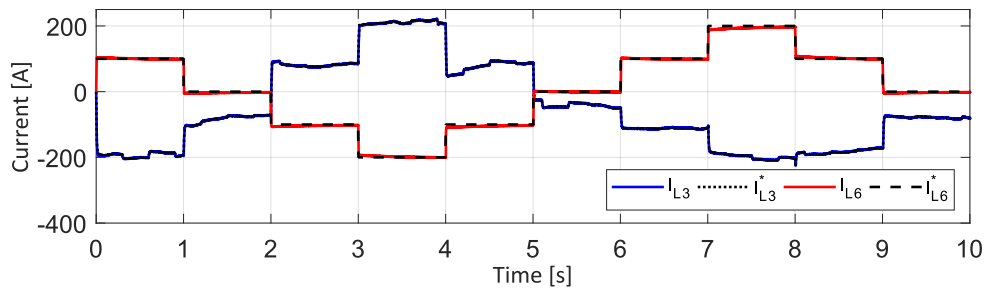

Figure 6. The current on the supercapacitor $I_{L_{3}}$ in blue and the current in the battery $I_{L_{6}}$ in red, following their references.

Figure 7 presents the controlled current $I_{L_{9}}$ on the PV system and its reference $I_{L_{9}}^{*}$. The chosen MPPT here is the incremental conductance one, which have fast tracking response compared with other algorithms. As the current is also influenced by the irradiation profile, its variations are quite similar to each other.

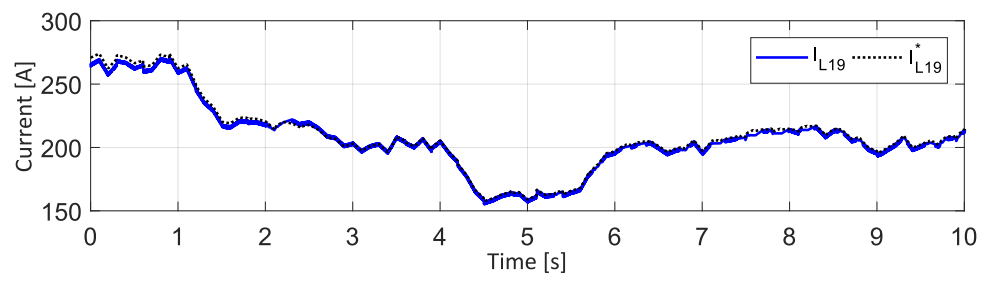

Figure 7. The controlled current on PV and its reference given by MPPT algorithm.

Figure 8 presents the demanded current in the DC load $I_{L_{13}}$ and its reference $I_{L_{13}}^{*}$. The current follows its reference allowing good control performance, then voltage is well 
controlled as consequence. The current here is negative to represent the power flowing from the DC bus to the load.

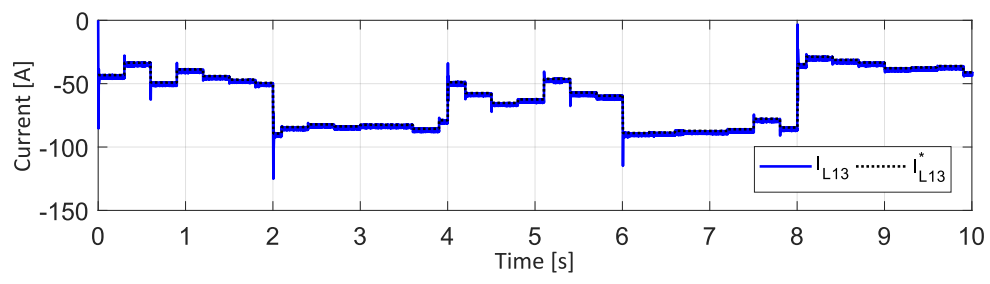

Figure 8. The current demanded by the DC load.

Figure 9 presents the output voltages converters that connects the devices (battery, PV and DC load) into the MicroGrid by the DC bus. They represent the zero dynamics of proposed control target, it is concluded that these variables present stable behavior.

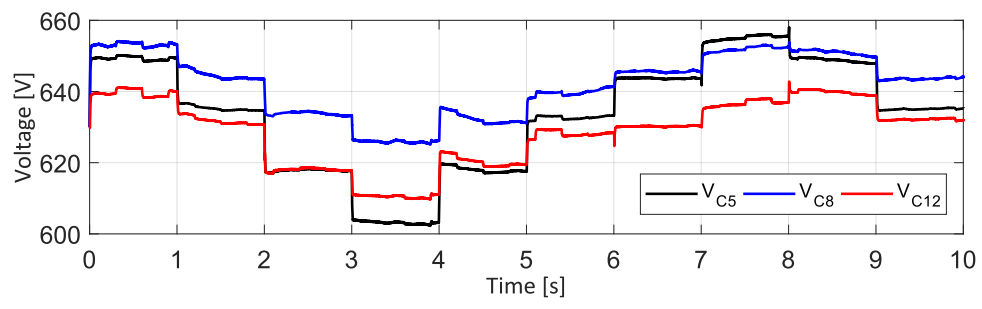

Figure 9. Output voltages on the MicroGrid converters (zero dynamics).

Figure 10 presents the voltage $V_{C_{2}}$ and its reference $V_{C_{2}}^{*}$, which is the desired voltage of the DC bus $V_{d c}$. Voltage $V_{C_{2}}$ is controlled according to the control target presenting fast control response with transient variations of $2 \%$ maximum error.

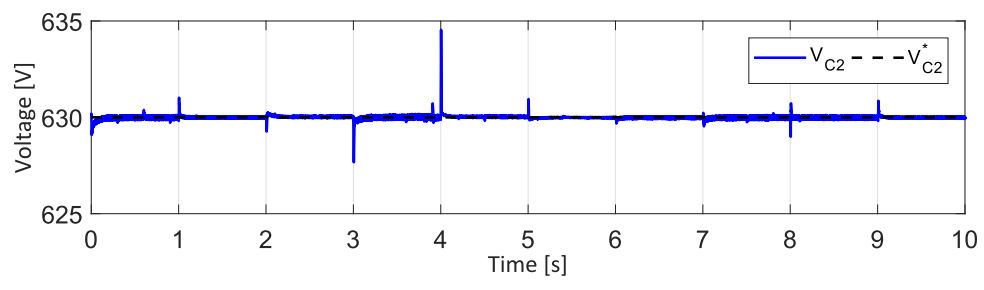

Figure 10. Voltage $V_{C_{2}}$ and its reference.

Figure 11 presents DC grid voltage $V_{d c}$ and its operation bounds. Voltage $V_{d c}$ is not controlled in a constant value, but is kept inside a desired region, which is given by the operation bounds of the electric grid (usually $\pm 5 \%$ ). In this case, voltage $V_{d c}$ varies according to the grid unbalance, and voltage $V_{C_{2}}$ is kept in the constant reference $V_{d c}^{*}$. The utilization of the fixed value $V_{d c}^{*}$ as desired reference for $V_{C_{2}}$ induces a steady state error in $V_{d c}$, given by the losses in $R_{2}$. Then, a smaller distance between the supercapacitor subsystem with the point considered for the desired DC bus voltage reduces the steady state errors on $V_{d c}$. 


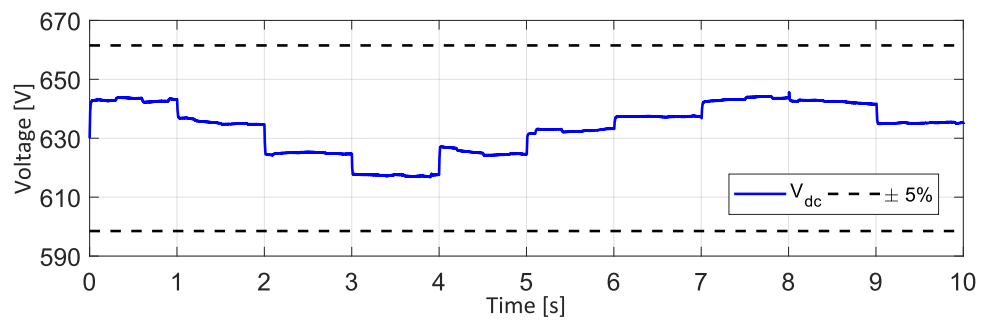

Figure 11. $D C$ voltage bus and the operation bounds.

\subsection{Comparison with PI control}

The linear PI controller is widely known and disseminated both in academia and in industry for its simplicity of application and robustness. However, in the case of electrical systems requiring better performance and greater energy efficiency, nonlinear control approach becomes a better fitted option. To validate this premise, a comparison of the proposed nonlinear control with the classic linear control approach was carried out in the developed MicroGrid.

Figure 12 presents a comparison on $V_{C_{2}}$ dynamics between a classical linear PI controller and the proposed nonlinear controller. It is clear that the PI response have much bigger overshoots in transients and a slower speed response. Therefore, the control performance of the nonlinear controller is better when compared with the PI controller.

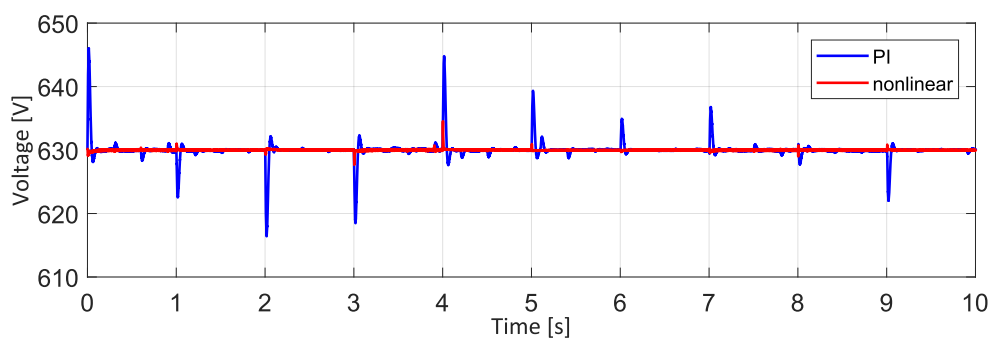

Figure 12. Comparison of $V_{C_{2}}$ dynamics by applying nonlinear control in red and classical Pl control in blue.

Figure 13 compares the different voltage behavior in the DC bus, using nonlinear controller (in red) and classical PI controller (in blue). The dynamics behavior for the nonlinear approach is faster with smaller overshoots during transients. For the PI control, the voltage $V_{d c}$ came close to surpassing the operating region of the system. In conclusion, the nonlinear control approach can stabilize MicroGrid's DC bus with better performance, which expose the linear control's limitations for a wider region of operation.

\section{Conclusions}

This paper proposes a nonlinear distributed control for an isolated DC MicroGrid composed of photovoltaic panels as the main generation; two energy storage system with different time-scales, a supercapacitor for fast variations and a battery for slow variations ; and a DC load that requires to be supplied with constant voltage. The load represent a generalization of the demanded power from the consumers. 


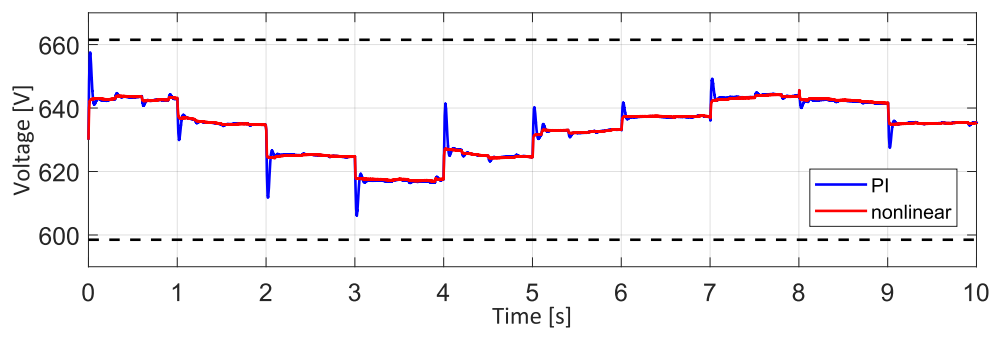

Figure 13. $D C$ bus voltage dynamics comparing the proposed nonlinear control and the linear PI controller.

The singular perturbation analysis based on time-scale separation in the supercapacitor system is well applied creating a stable control approach to stabilize the DC bus inside a desired region. Moreover, the control target of each device (PV, battery and DC load) is accomplished. The simulations results highlight the control performance of the MicroGrid and the comparison with the PI controller shows the better performance of the proposed nonlinear controller.

\section{Bibliography}

[1] J. G. De Matos, F. S. F. Silva, L. A. D. S. Ribeiro, « Power Control in AC Isolated Microgrids With Renewable Energy Sources and Energy Storage Systems », IEEE Transactions on Industrial Electronics, vol. 62, $\mathrm{n}^{\circ}$ 6, 2015.

[2] L. A. De Souza Ribeiro, O. R. SaAvedra, S. L. De Lima, J. G. De Matos, « Isolated Micro-Grids With Renewable Hybrid Generation : The case of Lençóis Island », IEEE Transactions on Sustainable Energy, vol. 2, $\mathrm{n}^{\circ}$ 1, 2011.

[3] L. A. De S. Ribeiro, O. R. SaAvedra, S. L. De Lima, J. G. De Matos, G. Bonan, «Making isolated renewable energy systems more reliable », Renewable Energy, vol. 45, 2012.

[4] R. H. LASSETER, «Smart distribution : Coupled microgrids », Proceedings of the IEEE, vol. $99, \mathrm{n}^{\circ} 6,2011$.

[5] L. E. Zubieta, « Are Microgrids the Future of Energy? : DC Microgrids from Concept to Demonstration to Deployment », IEEE Electrification Magazine, vol. 4, nº 2, 2016.

[6] J. J. Justo, F. Mwasilu, J. Lee, J. Jung, « AC-microgrids versus DC-microgrids with distributed energy resources : A review », Renewable and Sustainable Energy Reviews, vol. 24, 2013.

[7] D. Kumar, F. Zare, A. Ghosh, « DC Microgrid Technology : System Architectures, AC Grid Interfaces, Grounding Schemes, Power Quality, Communication Networks, Applications, and Standardizations Aspects », IEEE Access, vol. 5, 2017.

[8] F. Perez, A. Iovine, G. DAmm, P. Ribeiro, « DC microgrid voltage stability by dynamic feedback linearization », IEEE International Conference on Industrial Technology (ICIT), 2018.

[9] A. Iovine, S. B. Siad, G. Damm, E. De Santis, M. D. Di Benedetto, « Nonlinear Control of a DC MicroGrid for the Integration of Photovoltaic Panels », IEEE Transactions on Automation Science and Engineering, vol. 14, $\mathrm{n}^{\circ}$ 2, 2017.

[10] R. F. Bastos, T. Dragičević, J. M. Guerrero, R. Q. Machado, « Decentralized control for renewable DC Microgrid with composite energy storage system and UC voltage restoration connected to the grid », IECON - 42nd Annual Conference of the IEEE Industrial Electronics Society, 2016. 
[11] A. Iovine, S. B. Siad, G. Damm, E. De Santis, M. D. Di Benedetto, « Nonlinear control of an AC-connected DC MicroGrid », IECON - 42nd Annual Conference of the IEEE Industrial Electronics Society, 2016.

[12] W. Jing, C. Hung LAI, S. H. W. Wong, M. L. D. Wong, « Battery-supercapacitor hybrid energy storage system in standalone DC microgrids : areview », IET Renewable Power Generation, vol. 11, $\mathrm{n}^{\circ} 4,2017$.

[13] D. E. Olivares, A. Mehrizi-Sani, A. H. Etemadi, C. A. Canizares, R. Iravani, M. Kazerani, A. H. Hajimiragha, O. Gomis-Bellmunt, M. Saeedifard, R. PalmaBehnke, G. A. Jiménez-Estévez, N. D. Hatziargyriou, « Trends in Microgrid Control », IEEE Transactions on Smart Grid, vol. 5, n ${ }^{\circ}$ 4, 2014.

[14] L. Harnefors, A. G. Yepes, A. Vidal, J. Doval-Gandoy, « Passivity-Based Controller Design of Grid-Connected VSCs for Prevention of Electrical Resonance Instability », IEEE Transactions on Industrial Electronics, vol. 62, $\mathrm{n}^{\circ}$ 2, 2015.

[15] M. Zhao, X. YuAn, J. Hu, Y. YAn, « Voltage Dynamics of Current Control Time-Scale in a VSC-Connected Weak Grid », IEEE Transactions on Power Systems, vol. 31, n 4, 2016.

[16] Y. Chen, G. Damm, A. Benchaib, F. Lamnabhi-Lagarrigue, « Feedback linearization for the DC voltage control of a VSC-HVDC terminal », European Control Conference (ECC), 2014.

[17] P. Rouchon, « Necessary condition and genericity of dynamic feedback linearization », $J$. Math. Systems Estim. Control, vol. 4, n ${ }^{\circ}$ 2, 1994.

[18] B. Charlet, J. LÉvine, B. Marino, « On dynamic feedback linearization », Systems \& Control Letters, vol. 13, $\mathrm{n}^{\circ}$ 2, 1989.

[19] G. Oriolo, A. De LuCA, M. Vendittelli, «WMR control via dynamic feedback linearization : design, implementation, and experimental validation », IEEE Transactions on Control Systems Technology, vol. 10, $\mathrm{n}^{\circ}$ 6, 2002.

[20] R. MARINO, «Static and dynamic feedback linearization of nonlinear systems », Perspectives in control theory, 1990.

[21] S. X. Chen, H. B. Gooi, M. Q. WANG, « Sizing of Energy Storage for Microgrids », IEEE Transactions on Smart Grid, vol. 3, $\mathrm{n}^{\circ}$ 1, 2012. 